\title{
Le jeu du contrôle ou le contrôle du jeu : le discours direct et la participation dans l'interaction orale
}

\author{
Matthieu Monney ${ }^{1}$ \\ Université de Fribourg, Département de français
}

\begin{abstract}
Résumé. Si le discours rapporté direct (DD) est un dispositif commode pour (re)gagner le contrôle sur la parole ainsi que sur la présentation de soi et de l'autre, il apparaît en même temps comme un lieu privilégié de participation pour les auditeurs ou interlocuteurs. Le contrôle de la représentation se présente ainsi comme un enjeu capital de l'échange langagier, et l'on peut en distinguer deux modes de gestion opposés, que nous appelons la " chasse ouverte " et la "chasse gardée ». Ces configurations antagonistes de l'économie discursive et interactionnelle se jouent à la fois sur la circulation de la parole, sur la répartition des rôles communicationnels, sur la relation interpersonnelle ainsi que sur le mode de construction de la représentation.
\end{abstract}

\begin{abstract}
The game of control or the control of the game: direct reported speech and participation in spoken interaction. If direct reported speech is a convenient device in order to (re)gain control of speech and of presentation of self and other, it appears at the same time as a privileged spot for the auditors or partners to participate. The control of the representation therefore is at stake in the spoken exchange. Two modes of handling this control are to be distinguished : the " open ground », and the "preserve ». These configurations are resting at once on speech circulation, on the sharing out into communicative roles, on the interpersonal relationship and on the building mode of representation.
\end{abstract}

\section{Introduction}

La fréquentation régulière des transports publics a ceci de commode pour le linguiste qu'elle lui permet de travailler sans en avoir l'air : il lui suffit de regarder et d'écouter très attentivement ce qui se dit et la manière dont cela se dit. C'est par ce biais que nous avons pu remarquer un point commun entre les situations suivantes, parmi tant d'autres : un homme raconte ses déboires maritaux à un ami en faisant passer son épouse pour une parfaite harpie ; un employé rend de son patron un portrait à peu près stalinien ; une adolescente relate à quelque confidente une altercation avec ses parents, où ceux-ci passent pour les pires geôliers pour avoir fixé une heure de rentrée de toute évidence trop précoce. Le point commun est à chaque fois une intense utilisation argumentative du discours rapporté (DR), et notamment du discours rapporté direct (DD). Ce procédé a en effet souvent été décrit comme une ressource stratégique, un instrument qui permet au locuteur rapportant de « faire parler » (Ducrot, 1984 : 199) un locuteur rapporté, tout en feignant de s'effacer devant ce dernier, en lui laissant pour ainsi dire la parole, dans un « simulacre de non-intervention absolue » (Authier-Revuz, $1978: 51$ ). Cette impression relève toutefois 
bien d'une feinte et d'une mise en scène. Le DD se présente en réalité comme une « construction » (Marnette, 2002) du rapporteur, l'énoncé étant « appropriated » (Tannen, 1989 : 101) dans les deux sens du terme : (i) le rapporteur fait sien l'énoncé rapporté ; et (ii) il l'apprête ou l'accommode pour un usage particulier, dans l'interaction actuelle. Comme le rappelle Marnette ${ }^{2}$ (2002: 8), "'reprendre', comme 'rapporter', c'est toujours changer de contexte et utiliser le discours de l'autre de manière différente, à la limite opposée de ce qu'il a pu ou pourrait dire ». Les principaux mécanismes constituant cette opération complexe d'appropriation sont les suivants :

(i) De la façon la plus évidente, la séquence rapportée (SR), en tant que telle, subit une décontextualisation et une recontextualisation, ce qui tient à son insertion à la fois dans une situation d'énonciation autre et dans une trame discursive autre. Or c'est ce nouvel environnement qui fonctionne comme le cadre d'interprétation décisif de la citation ${ }^{3}$.

(ii) L'appropriation du discours tient ensuite au fait que c'est le rapporteur qui (re)construit la matière verbale de la citation - c'est-à-dire qui procède à sa textualisation, par différentes opérations de « découpage-collage » (Vincent \& Dubois, $1995: 321)$ par lesquelles la parole autre se trouve aménagée pour les besoins du rapporteur, selon un principe que Vincent (2004:238) rapproche de celui du « best of ».

(iii) Le rapporteur est également responsable de la mise en voix de la citation, et donc de sa matière paraverbale, qui peut apparaître comme plus ou moins imitative ou autre contraire décalée par rapport à celle - supposée, imaginée ou inférée - de la citation originelle ${ }^{4}$.

(iv) Un dernier élément qu'il faut mentionner, et qui pèse de manière déterminante sur l'appropriation d'un discours autre, tient à la très fréquente absence du locuteur rapporté ou à son manque d'emprise sur la mise en scène dont il fait l'objet ${ }^{5}$. Or les absents ont ceci de commode qu'ils ne sont plus les «déités » (Goffman, 1982 : 95) ou les « objets sacrés » (ibid. : 91) des interactions en présence mutuelle, et donc qu'ils échappent à l'exigence de politesse, aux précautions et aux égards, ou aux " protectives practices » qui définissent en partie le «face-work » (voir Goffman, 1982). De ce fait, les absents se présentent aux interactants comme une matière aisément malléable, comme des sortes de marionnettes que ces derniers font bouger et parler pour leurs propres visées dans l'interaction effective. Évidemment, les ficelles qui trahissent le marionnettiste sont plus ou moins grossières. Mais même dans les cas où le locuteur rapporté est également un interlocuteur effectif, il n'est pas forcément évident de résister à l'aliénation que produit la mise en scène de soi par un autre, car cela exige d'intervenir au moyen de corrections, remodèlements, contestations, etc. qui sont autant d'actes de langage coûteux en énergie et en menace potentielle sur les faces en présence.

Considérant les moyens d'appropriation définitoires du DD, ce dispositif apparaît comme un procédé rhétorique aux rendements discursifs multiples, grâce à la conjonction de la grande plasticité verbale et mimo-gestuelle des énonciations « rapportée », et de la relative illusion d'indépendance dont jouissent ces représentations. C'est ce que souligne Tuomarla (2000 : 173) en affirmant que le DD permet essentiellement de « mieux communiquer et mieux se cacher ». À bien des égards, son fonctionnement pourrait être décrit, toutes proportions gardées, comme une forme de « let's take back control », d'après le slogan des proposants du Brexit. Or la question du contrôle de la représentation et de la parole dans l'interaction, en lien avec la co-construction des DD, sera justement un objet central dans notre analyse. Il s'agira plus précisément de saisir la tension entre l'appropriation du DD par un locuteur et les possibilités de désappropriation, de perte de contrôle pouvant survenir par l'inscription de la représentation dans un échange, impliquant des interlocuteurs qui ne se laissent pas réduire à de simples réceptacles. 
L'analyse conversationnelle l'a bien saisi : l'occupation par plusieurs interlocuteurs d'un fil de parole unique ${ }^{6}$ nécessite une organisation relativement réglée, une synchronisation et une coordination permanente entre eux. C'est-à-dire que le discours est forcément une activité collaborative des interactants, où le flux communicationnel émergent dépend de la «participation $»^{7}$ qui se met en œuvre. En effet, comme le démontrent Charles et Marjorie Goodwin, «the process of creating a participation framework in which speaker and hearer are aligned to each other can shape, and be shaped by, the detailed organization of the talk produced within that participation framework » (2004: 230). Toutefois, l'ajustement des participants entre eux et par rapport à l'interaction en cours ne fait pas forcément de la parole un espace d'harmonie pacifiée. Il implique seulement ce trait fondamental de tout événement co-construit, à savoir « a distributed responsibility among interlocutors for the creation of sequential coherence, identities, meaning, and events » (Jacoby \& Ochs, 1995 : 177). Mais l'espace de parole s'avère au contraire bien souvent, en raison précisément des identités et des sens qui y sont mis en jeu, un espace de tension, un territoire sans cesse à renégocier. Nous examinerons à partir de là, sur le champ de la coconstruction des DD, deux façons opposées «d'être à plusieurs » (Flahault, 2013), à travers l'interrelation des trois espaces d'insertion du sujet (EIS) de Charaudeau (1994). Ceux-ci se définissent en rapport aux trois problèmes auxquels doit répondre le sujet parlant pour entrer dans un échange : (i) comment occuper l'espace de parole ? (ii) comment se positionner par rapport aux autres intervenants ? et (iii) comment se positionner par rapport au contenu des propos tenus? À chacun correspond respectivement un EIS spécifique, qui sont : (i) l'espace de locution ou d'interlocution, (ii) l'espace d'influence ou de relation, et (iii) l'espace de thématisation. Nous verrons se dessiner deux économies, deux modes bien distincts de gestion de l'interaction, qui seront désignés l'un comme la " chasse ouverte », l'autre comme la «chasse gardée ». Pour ce faire, nous analyserons pour chacun un extrait de conversation représentatif de ces types. Les extraits en question proviennent du corpus OFROM, et il est possible de les écouter sur le site http://www11.unine.ch/, au moyen par exemple des références indiquées en annexe.

\section{Chasse ouverte}

Dans l'interaction qui nous occupera pour ce chapitre, deux jeunes femmes partagent leurs expériences d'enseignantes dans des classes d'enfants. Dans la transcription que nous en avons effectué, les soulignements en trait continu correspondent aux segments de DD, et les soulignements en pointillé aux segments de discours intériorisé (DInt). Le reste de nos conventions de transcription est disponible à la suite de la bibliographie. Voici l'extrait en question :

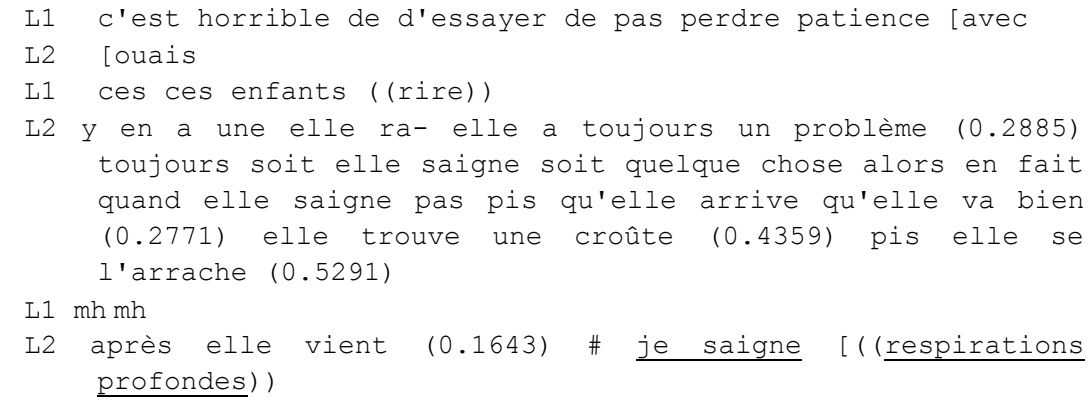




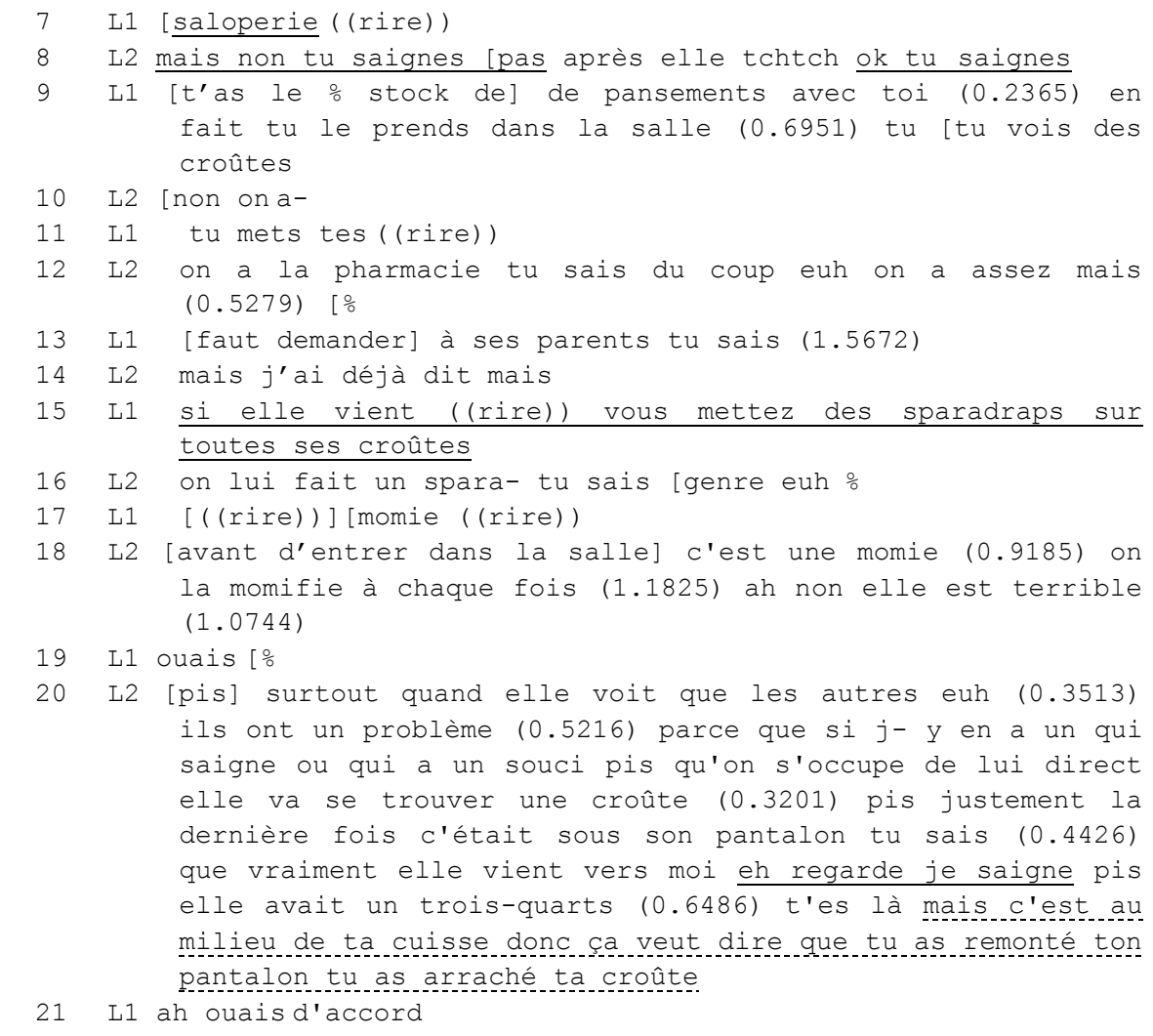

Sous la forme d'une exemplification, introduite par le présentatif y en a une au tour de parole (TP) 4, L2 présente une de ses élèves, sur la base d'un comportement récurrent (l'adverbe de fréquence toujours au TP 4). La scène d'interaction rapportée vient incarner un échange typique auquel il donne lieu, tout en permettant d'élaborer la caractérisation de cette élève. Le premier DD au TP 6, amené par une séquence introductive (SI) construite autour d'un verbe de mouvement (vient), surjoue intonativement la détresse de l'enfant. La réponse de l'enseignante, en l'occurrence L2 en tant que personnage, est élaborée sur plusieurs interventions : L2 présente d'abord elle-même, au moyen du DD, sa propre réaction en trois temps : (i) une série de respirations profondes, mimant sa tentative de rester calme face à une situation stressante ou exaspérante (ce qui constitue bien, en tant qu'action communicative, une forme de DD, dans une conception mimique de celui-ci : $c f$. de Cornulier, 1978 et Gachet, 2015) ; (ii) une dénégation de l'affirmation de l'élève (mais non tu saignes pas); et (iii) une rétractation (ok tu saignes), faisant suite à l'onomatopée tchtch mimant un jaillissement de sang. Divers moyens et canaux sémiotiques se trouvent mobilisés pour la création d'une scène fortement dramatisée, en entrelaçant des ressources à la fois verbales et paraverbales, qui donnent à voir, sentir et entendre les événements et personnages représentés.

Mais dans le même temps, en chevauchement sur la première intervention rapportée de L2 (la série de respirations profondes), L1 quitte son rôle de simple auditrice (dont est caractéristique notamment le continuateur $m h m h$ au TP5) et entre en scène, en se projetant à la place du personnage L2 et en jouant son rôle. Elle présente avec le DR saloperie une réaction imaginée de $\mathrm{L} 2$, concurrente ou complémentaire par rapport à celle construite par L2 elle-même. Ce DR relève selon nous d'un DInt, étant donné d'une part la faible probabilité qu'une telle intervention soit prononcée à haute voix dans la situation décrite, et d'autre part l'intonation chuchotante affectée à l'intervention. L1 participe ainsi à la représentation du personnage L2, en s'arrogeant au même titre que L2 le rôle de locutrice rapportante. Sa contribution, comme nous l'avons indiqué, peut s'analyser selon deux 
logiques distinctes : celle du patchwork, suivant un principe de complémentarité ou de cumulation ; et celle du palimpseste, suivant un principe de concurrence ou de contradiction.

La participation de L1 au récit de L2 s'accroît ensuite, entre les TP 9 et 17. C'est même elle qui prend pour un temps la mainmise sur la narration, en dirigeant son déroulement et en allant jusqu'à faire émerger de nouveaux personnages, les parents de l'élève, auxquels s'adresse un nouveau DD attribué à L2 au TP 15. Cet investissement se traduit par de nombreux chevauchements, par une domination quantitative et qualitative de l'espace de parole de la part de L1, par son emprise sur l'introduction d'objets-de-discours dans le fil narratif, et par le DD qui fait parler le personnage L2 au TP 15. Depuis la première incursion de L1 au TP 7 jusqu'au TP 17, on assiste ainsi à l'insinuation croissante de L1 dans le récit de L2 : d'un DInt suggéré (saloperie), sans effet notable sur la narration de L2, L1 s'empare progressivement du territoire discursif et narratif, à travers un éloignement progressif de la scène représentée de départ, au moyen de scènes inventées. En effet, en investissant une histoire dont elle n'est pas une participante, L1 ne peut procéder que par projection ou identification et par développements imaginés.

Face à cette forme de " colonisation » de son propre récit, on perçoit chez L2 une évolution stratégique ${ }^{8}$ dans sa réaction. Dans un premier temps, elle tente de maîtriser ces incursions, en réagissant dans les TP 10, 12 et 14 aux développements de L1 par une argumentation construite sur la dénégation (non on a- [...] on a la pharmacie tu sais du coup euh on a assez mais) et le démenti de sous-entendus (mais j'ai déjà dit mais). La tentative de reprise de son récit par L2 apparaît en même temps comme une tentative de le réancrer dans la réalité (une forme de " ça s'est passé comme ci et pas comme ça »), dont elle seule est la détentrice pour le cas présent. Mais dans un deuxième temps, aux TP 16 et 18, L2 semble se rallier au tour pris par la narration, en quittant une posture de maîtrise et de résistance, pour adopter davantage une posture d'assentiment et de connivence. Par-là, elle entre en quelque sorte dans le jeu de L1, en participant à l'élaboration fictionnelle du récit et en prenant à son compte certains des éléments introduits par cette dernière, tels que les sparadraps ou l'image de la momie. Cette manœuvre quasiment concessive débouche même en fin de compte sur le rétablissement de l'asymétrie initiale des rôles communicationnels entre L2 narratrice et L1 auditrice.

À ce stade, deux remarques sont utiles : premièrement, il a été affirmé dans l'analyse conversationnelle que le récit allait de pair avec la suspension négociée de l'alternance des TP (voir par exemple Jefferson, 1978). Or notre extrait montre bien que ce n'est pas forcément le cas et que l'on trouve également des narrations co-construites par les interventions de plusieurs locuteurs, avec une alternance voire un brouillage plus ou moins important des rôles communicationnels de narrateur et d'auditeur' ${ }^{9}$. Deuxièmement, l'extrait rappelle bien la nécessité de dépasser une vision égocentrée de l'énonciation, celle de la polyphonie de Ducrot (1984) ou du footing de Goffman (1981) par exemple ${ }^{10}$. Les deux chercheurs se concentrent, dans leur contestation de "l'unicité du sujet parlant » (Ducrot, 1984 : 171), sur des distinctions au sein de segments relevant d'un seul locuteur. Or dans notre exemple, L2 et L1 participent conjointement, notamment par différentes formes de DR, au « faire parler » d'un même locuteur rapporté ou personnage. Dans les termes de Goffman, il apparaît qu'une seule « figure $»^{11}$ est co-construite par deux « animators $»^{12}$. L'analyse du théâtre qu'organise la parole des locuteurs, pour reprendre la célèbre métaphore ducrotienne, doit tenir compte de la dimension interactionnelle de la parole et donc de la possibilité de voir un même personnage interprété alternativement ou même simultanément par plusieurs acteurs. À partir de là va forcément se poser la question de la cohérence de l'entité ainsi construite. Dans notre cas, si l'on se concentre sur le chevauchement entre les TP 6 et 7, on perçoit une tension dans l'ethos ${ }^{13}$ du personnage L2, tel que le donne à voir les DR des deux interlocutrices : un être qui parvient malgré tout à garder son calme dans le DD de L2 (les respirations profondes), ou qui ne contient pas vraiment son irritation, dans le DInt de L1 (saloperie). Il serait intéressant d'approfondir ce 
mode de représentation à deux voix, en examinant par exemple le statut de chaque contribution, son degré de légitimité, son rôle narratif ou argumentatif, et d'autres facteurs encore. Nous y reviendrons quelque peu dans le chapitre suivant.

$\mathrm{Au}$ vu de ces observations, ce que nous appelons la « chasse ouverte » se définit comme une configuration particulière de l'économie interactionnelle et de la participation, caractérisée par l'articulation des traits suivants, au sein des trois EIS : l'espace d'interlocution (i) voit s'instaurer une circulation assez libre de la parole ainsi qu'une relative interchangeabilité des rôles communicationnels, ce qui se traduit par la multiplication des chevauchements, par la tendance au raccourcissement des interventions, ainsi que par une répartition relativement égale du temps de parole ; l'espace d'influence (ii) se construit sur des relations plutôt symétriques, où l'accent est mis sur la coparticipation, la connivence, voire même la communion ${ }^{14}$ entre les interactants. Dans ce cadre, c'est d'abord le besoin psychologique que Lipiansky (1993: 33) appelle « d'intégration » qui nous semble concerné, et qui est décrit comme le fait d' « être jugé positivement, [de] donner une bonne image de soi, [d']être apprécié... » (ibid.). Pour ce qui concerne enfin l'espace de thématisation (iii), c'est-à-dire le positionnement par rapport au contenu des propos tenus, il est caractérisé par un départ de la réalité ou du moins par un rapport plus lâche à celle-ci, qui s'ouvre aux ajouts et développements fictionnels, aux dérivations imaginées, en un mot aux mondes possibles que le discours peut susciter. Cet espace recoupe en partie le concept de la "mémoire discursive» dans le sens de « l'ensemble évolutif de représentations publiquement partagées qui s'élaborent [...] coopérativement au long d'un discours » (Groupe de Fribourg, 2012 : 22). Dans la chasse ouverte, la mémoire discursive construite par le récit s'ouvre donc d'une part aux contributions des diverses participantes, sur un mode cumulatif et collaboratif, et d'autre part à la fiction ou à l'imagination, par des élaborations et dérivations sur une base d'expériences vécues. Bien entendu, ces différents traits dans les trois EIS se soutiennent mutuellement.

Concernant le contrôle, la chasse ouverte, en tant que configuration négociée, peut se décrire ainsi : l'initiateur du récit cède du terrain quant au réalisme du récit, à la maitrise de son déroulement et à la domination de l'espace de parole, en vue de favoriser une coconstruction participative de scènes ouvertes aux exagérations, dérivations en cascade, inventions et autres modulations fictionnelles. Le récit devient une sorte de terrain de jeu, dans lequel chaque interactant peut devenir tour à tour acteur, dramaturge et metteur en scène. Notons bien toutefois que ce ne sont pas tant les narrations en soi qui relèvent de la chasse ouverte ou gardée, mais plutôt des " moments " de leur déroulement où de telles configurations émergent dans une négociation sans cesse reprise de la " définition de la situation ", pour reprendre la problématique goffmanienne au cœur de The Presentation of self in everyday life (1959).

\section{Chasse gardée}

Nous examinerons ici la configuration interactionnelle et narrative opposée à la précédente, et que nous avons appelée la « chasse gardée ». Pour ce faire, nous étudierons successivement deux extraits d'une même conversation entre trois amis, deux hommes et une femme. L'échange porte sur l'achat d'un lit par l'un des interactants.

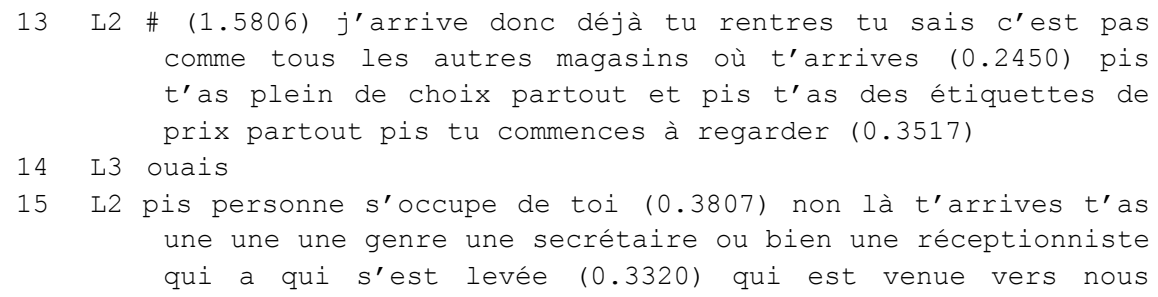




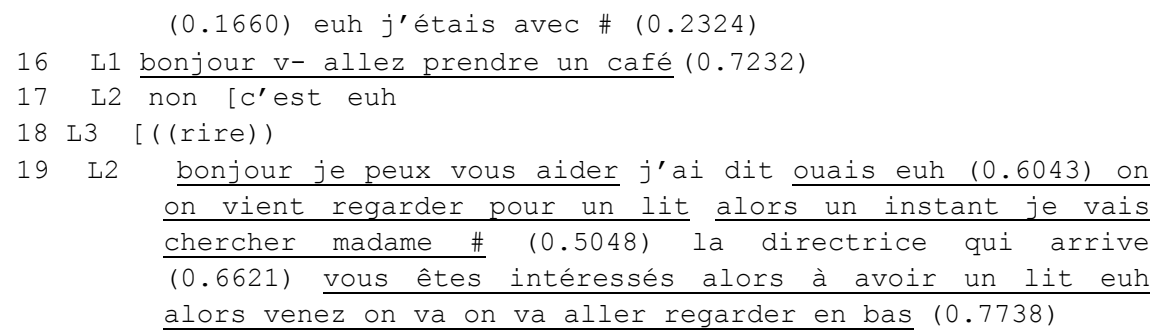

Ayant au préalable mentionné le nom du magasin de meuble, L2 relate son arrivée dans l'établissement. Il assure la " racontabilité ${ }^{15}$ de son récit au moyen d'une mise en scène contrastive : c'est pas comme dans les autres magasins... (comparaison niée) - non là t'arrives... Le mouvement se construit sur un TU générique, qui va donner place aux JE et NOUS singularisés. L2 représente ainsi son expérience comme littéralement hors du commun. Puis il introduit le personnage de la secrétaire ou réceptionniste dans une séquence ( $t$ 'as une une une genre une secrétaire ou bien une réceptionniste qui a qui s'est levée (0.3320) qui est venue vers nous, TP 15) que l'on peut analyser comme une introduction de DD (une SI). Cette interprétation s'appuie d'une part sur la fréquence des SI en forme de description d'un rapprochement entre deux ou plus interlocuteurs potentiels, qui peuvent même souvent se passer de verbum dicendi; et d'autre part sur l'inscription de cette scène dans un script commercial, où l'on attend du vendeur se dirigeant vers les clients qu'il leur propose son assistance.

Mais au moment où L1 anticipe le DR de L2, juste après que ce dernier justifie de façon parenthétique le passage du singulier (le JE et le TU) au pluriel (le NOUS) dans euh j'étais avec \# (TP 15), L1 s'immisce dans le récit en produisant lui-même un DD attribué à la réceptionniste. Ce faisant, il coupe en quelque sorte l'herbe sous les pieds de L2, en surenchérissant sur la description de la réceptionniste par ce dernier, par le fait d'exagérer comiquement (voir le ((rire)) de L3 au TP 18) la proposition d'assistance attendue en proposition de consommation ( $v$-allez prendre un café). Le DD en question, dans la classification de Vincent \& Dubois (1997), est en tension entre la " reproduction », dans le sens où il existe une situation source passée, avec des interlocuteurs déterminés et une situation de communication plausible, et $l^{\prime}$ « invention $»^{16}$, qui tient à l'improbabilité du contenu du DD, due à l'exagération humoristique. Par son incursion, L1 dans le même temps empiète sur le territoire de parole de L2, s'arroge l'effet que celui-ci projetait en s'insérant à un endroit stratégique du récit, et fait violence à la tonalité réaliste de ce dernier en le tirant vers la caricature ou la parodie.

Dans un cas de chasse ouverte (supra, § 2), L2 pourrait alors baisser la garde et embrasser cette inflexion en surenchérissant à son tour, par exemple. Mais sa réaction est bien différente. Il mitige l'intervention de L1 d'abord par une négation (non), puis une rectification (c'est euh...), pour introduire un DD plus approprié à la situation, c'est-à-dire dont le contenu même relève de la "reproduction » (voir supra), et qui se substitue à l'exagération de L1 et rétablit la tonalité plutôt réaliste du récit. Après cela, L2 poursuit sa narration par un rapport d'interaction, comme si de rien n'était : l'invasion barbare est repoussée, les frontières sont rétablies, l'histoire reprend son cours. Le mode d'alimentation de la mémoire discursive n'apparaît plus tant cumulatif et symétrique, comme dans la chasse ouverte, que concurrentiel et asymétrique. Cela se traduit notamment par des dissensions dans la construction de l'image du personnage, dont les paroles attribuées par les deux locuteurs peignent un ethos discursif décousu, incohérent, voire contradictoire.

Un deuxième extrait de la conversation est très représentatif de l'économie interactionnelle de la chasse gardée. Il survient après que L2 a annoncé à la directrice, dans son récit, le prix qu'il pensait mettre pour un lit complet, à savoir mille deux cents francs. 


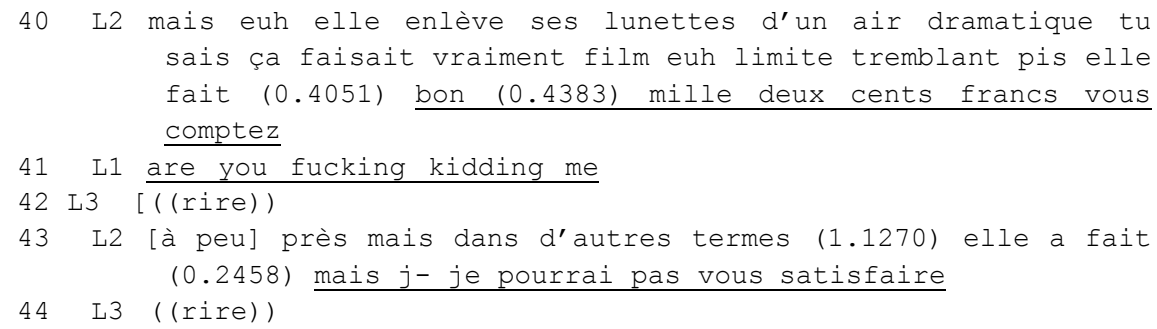

Il s'agit là d'un épisode clé, étant donné qu'il porte sur la question de l'argent, tendanciellement délicate dans l'échange commercial, étant donné les menaces potentielles sur les faces des interactants. La réaction de la directrice est présentée en trois temps : tout d'abord, dans le TP 40, L2 en construit un mime fortement dramatisé, au moyen d'une SI thématisant la théâtralité de la scène (d'un air dramatique, juxtaposé à ça faisant vraiment film euh limite tremblant) et d'une SR (bon (0.4383) mille deux cents francs vous comptez), dans laquelle la directrice répète la somme indiquée par le personnage L2, en l'introduisant par un atténuateur intersubjectif bon ${ }^{17}$, pour amorcer ensuite une évaluation (vous comptez), moment où arrive une nouvelle interruption de L1. Jusque-là, L2 représente efficacement l'embarras désolé de la directrice, prise en double bind entre la nécessité d'informer ses clients de l'insuffisance du montant, tout en ménageant leur face et la sienne. L'intonation ${ }^{18}$ attribuée par L2 participe largement de la dépiction de cette attitude, à travers une baisse marquée de l'intensité sonore - quasiment jusqu'au chuchotement - et de la hauteur mélodique, un ralentissement du rythme et un ton de voix très contenu.

Dans un deuxième temps arrive, comme annoncé, l'incursion en DD de L1 en are you fucking kidding me (TP 41). Celle-ci oscille à nouveau entre la "reproduction » et l'« invention »: d'un côté il y a bien une scène d'énonciation représentée identifiable et concrète, mais de l'autre, l'énoncé est en rupture manifeste avec le style communicatif et l'ethos de la directrice, tels que représentés par L2. L'écart porte à la fois sur l'intonation, le positionnement interpersonnel, le registre de langue, et la langue même. En effet, L1 mime une réaction agressive, à travers une exclamation stéréotypique ${ }^{19}$ d'exaspération et d'incrédulité, vulgaire et en anglais ${ }^{20}$, dont le rythme rapide et saccadé ainsi que l'intonation basse et contenue donnent une impression d'irritation froide. Ce faisant, L1 propose un mime ostensiblement outrancier, à teneur comique. Il est analysable soit comme un véritable $\mathrm{DD}$, faisant concurrence à l'ethos poli et légèrement affecté attribué à la directrice par L2, soit comme un DInt, représentant une réaction intérieure dont le contraste ethotique tiendrait à la différence entre ce qui peut être pensé (are you fucking kidding me) vs ce qui peut être dit (bon (0.4383) mille deux cents francs vous comptez). 
La réaction de L2 au TP 43, en dernier lieu, consiste en une concession (à peu près mais dans d'autres termes), qui débouche sur une reformulation en DD de l'évaluation de la directrice (la SI elle a fait, et la SR mais $j$ - je pourrai pas vous satisfaire), dans un style congruent avec son ethos tel que représenté par L2, et approprié à la situation, c'est-à-dire de distance polie. À nouveau, L2 parvient à garder la mainmise sur son récit et à gérer les « definitional disruptions » (Goffman, $1990: 24$ ), en filtrant ce qui peut y entrer et la façon dont cela participe à son élaboration, au moyen de stratégies diverses de mitigation, de concession, de négation, et/ou de reformulation. Dans le cas que nous venons de voir, il traite l'intervention de L1 attribuée à la directrice comme « ce qu'elle aurait pu penser mais n'a pas pu dire ", ce qui lui permet ensuite de la remplacer par un DD correspondant selon toute vraisemblance davantage à ce qui a pu être exprimé.

Si l'on revient aux trois EIS, en guise de synthèse, l'économie interactionnelle et discursive de la « chasse gardée » se caractérise, pour l'espace d'interlocution (i), par une gestion différenciée et hiérarchisée des rôles communicationnels, assortie à une répartition très inégale de la parole. L'espace d'influence (ii) est structuré sur une relation asymétrique entre les interactants : le narrateur garde l'emprise sur son récit en attendant de ses interlocuteurs une attitude plutôt passive de spectateur ou d'auditeur, et en traitant les incursions de ces derniers comme des menaces potentielles à désamorcer, à la manière dont pourrait le faire un organe de censure ou de propagande. L'intervention de l'auditeur dans le récit apparaît alors comme une profanation, une façon de ne pas rester à sa place. Les besoins psychologiques visés par le narrateur, toujours dans la typologie de Lipiansky (1993 : 33), tendent davantage vers la « valorisation », (« être jugé positivement, donner une bonne image de soi, être apprécié... ») et le "contrôle » (" pouvoir maîtriser l'expression et l'image que l'on donne de soi »). Finalement, l'espace de thématisation (iii) voit les frontières du récit jalousement gardées par le narrateur, qui ne laisse que peu la narration sortir des rails que lui-même a posées, en rétablissant l'ordre à chaque fois qu'une disruption possible émerge. Il faut bien sûr à nouveau envisager ces traits en termes d'interdépendance.

Pour ce qui concerne le contrôle, le locuteur principal cette fois ne lâche rien, ou pas grand-chose. Il adopte une posture que l'on pourrait quasiment définir par cette paraphrase de la célèbre déclaration christique : «C'est moi qui suis le chemin, la vérité et la vie. On ne vient au Récit qu'en passant par moi. »C'est-à-dire que la mémoire discursive est gérée de manière très asymétrique, étant donné qu'elle se trouve aux mains d'un seul locuteur, qui en est le premier investisseur ainsi que le gardien.

\section{Conclusion}

$\mathrm{Au}$ terme de ce parcours, on constate que l'espace du récit peut se trouver investi de façons très différentes, donnant lieu à des modes de participation ainsi qu'à des économies interactionnelles et discursives opposées sur le plan de la répartition des rôles communicationnels, de la gestion du fil de la parole, du rapport au réel et de la construction de la mémoire discursive, ainsi que des positionnements mutuels des participants et de la relation interpersonnelle. Ces "moments » de l'interaction peuvent se résumer par un rapport distinct au contrôle : du côté de la chasse ouverte, le narrateur concède des droits d'accès et de direction du récit, en vue de gains sur la relation interpersonnelle. Celle-ci est valorisée en raison de la relative symétrie instaurée par une telle économie de l'échange, où l'espace du récit devient un terrain de jeu ; du côté de la chasse gardée, le narrateur s'accroche à ses droits et au récit en tant que son espace privilégié, ce qui en fait pour les interlocuteurs une forme de spectacle auquel ils assistent, en manifestant régulièrement leur attention voire leur approbation, mais pas davantage. Ces deux configurations sont évidemment des types, qui peuvent s'instancier sous des formes multiples et à des degrés divers. Nous avons sciemment sélectionné des extraits très représentatifs et clairs, mais il faut bien noter d'une part que la répartition entre chasse gardée et chasse ouverte n'est pas 
toujours évidente, et d'autre part que l'on peut passer insensiblement d'une économie à l'autre assez rapidement et fréquemment.

En raison de leurs caractéristiques respectives, ces économies favorisent certains emplois distincts du DD dans la typologie de Vincent \& Dubois (1997 : 59-64) : la chasse ouverte tendra davantage vers la «pseudo-reproduction » et l' « invention », alors que la chasse gardée se situera plutôt dans la « reproduction » et l' « actualisation ». Ces emplois répondent logiquement à l'organisation discursive et interactionnelle plus générale de l'échange, en même temps qu'ils contribuent à l'instaurer et à la négocier.

Pour conclure, il nous a semblé, en examinant de nombreux échanges en plus des deux présentés ici, que la participation par le DR prenait le plus souvent la forme du DD. Ce penchant pourrait s'expliquer selon nous par les éléments suivants définissant le dispositif du DD : celui-ci se caractérise par une relative indépendance énonciative (i), l'espace de la SR apparaissant comme distinct et autonome (même si cela relève comme nous l'avons rappelé d'un simulacre). De ce fait, les interventions rapportés se distancient en quelque sorte de leur locuteur, étant attribuées à un énonciateur autre, ou relevant du moins d'une énonciation autre; ce qui favorise ainsi leur investissement par un interlocuteur. Ensuite, le DD tend à l'indépendance syntaxique (ii), étant du côté de la juxtaposition, de la parataxe ou du collage (là où le discours indirect relève plutôt de la subordination et de l'intégration). Or cela facilite la participation, en raison d'une part de la dissociation a priori plus importante des composants de l'énoncé (notamment entre la SI et la SR), et d'autre part de la simplicité de la textualisation d'un DD, qui tient à l'absence du type de calcul de concordance de temps et de personne qu'implique le DI. La disjonction entre les constituants dans un énoncé contenant un DD est redoublée par la rupture sémiotique (iii) que celui-ci engage, à savoir le passage de l'« usage " à la " mention » (Authier-Revuz, 1978, 2000, 2004), ou de l' « acte linguistique » à l'« acte mimique » (de Cornulier, 1978). Le Grammaire de la période (2012 : 68) traite d'ailleurs le DD comme un " collage intersémiotique ». Le dernier facteur favorisant le choix du DD correspond selon nous à l'intérêt dramatique et rhétorique (iv) de ce dispositif, dû à l'illusion d'indépendance qui le sous-tend ainsi qu'aux possibilités de jeu sur la mise en voix, sur l'incarnation qu'il permet. Caillat en parle même comme d'un « changement de peau » (2012 : 116). Il apparaît donc qu'à peu près tout ce qui rend le DD commode pour la participation est en même temps, sous une autre perspective, ce qui en fait un dispositif propice à l'argumentation ainsi qu'à la présentation de soi et de l'autre. Reste donc à savoir, pour chaque cas, si le jeu en vaut la chandelle.

\section{Références bibliographiques}

Amossy, R. (2010). La Présentation de soi. Ethos et identité verbale. Paris : Presses Universitaires de France.

Authier-Revuz, J. (1978). Les formes du discours rapporté. Remarques syntaxiques et sémantiques à partir des traitements proposés. DRLAV, 17, 1-87.

Authier-Revuz, J. (2000). Le Fait autonymique : langage, langue, discours - quelques repères. Dans Actes du colloque : le fait autonymique dans les langues et les discours [en ligne]. SYLED, Université de la Sorbonne Nouvelle, Paris, 5-7 octobre 2000, URL : http://syled.univparis3.fr/colloques/autonymie-2000/theme1/authierrel.pdf, page consultée le 17 avril 2019. 
Authier-Revuz, J. (2004). La Représentation du discours autre : un champ multiplement hétérogène. Dans Munoz, J. M., Marnette, S., Rosier, L. (éds). Le Discours rapporté dans tous ses états. Actes du Colloque International, Bruxelles, 8-11 novembre 2001, 35-53.

Beeching, K. (2007). La co-variation des marqueurs discursifs bon, c'est-à-dire, enfin, hein, quand même, quoi et si vous voulez : une question d'identité ? Langue française, 154, 79-93.

Caillat, D. (2012). Le discours représenté direct dans les conversations orales : une gestion multimodale des décrochements énonciatifs. Le Discours et la langue [en ligne]. 113-122.

Caillat, D. (2013). Le traitement multimodal des discours rapportés (en style direct) à l'oral : procédés énonciatifs et argumentatifs du recours aux émotions. Semen [en ligne], 35, URL : https://journals.openedition.org/semen/9816\#tocto2n2, page consultée le 17 octobre 2018.

Charaudeau, P. (1994). Les espaces de parole dans la situation d'interlocution. Cahiers $d u$ Centre Interdisciplinaire des Sciences du Langage, 10, 335-345.

Corpus Oral de Français de Suisse Romande (OFROM) [en ligne]. Université de Neuchâtel, URL : http://www11.unine.ch/, page consultée le 6 janvier 2020.

Ducrot, O. (1984). Le Dire et le dit. Paris : Minuit.

Flahault, F. (2013). Une manière d'être à plusieurs. INfluencia [en ligne]. Hors-série, URL : http://influencia.net/larevue/hors-serie-conversation/index.php?page=6, page consultée le 6 décembre.

Goffman, E. (1981). Forms of talk. Philadelphia : University of Pennsylvania Press.

Goffman, E. (1982 [1967]). Interaction ritual. Essays on face-to-face behavior. New York : Pantheon.

Goffman, E. (1990 [1959]). The Presentation of self in everyday life. London : Penguin Books.

Goodwin, C., Goodwin, M. H., (2004). Participation. In Duranti, S (ed.). A Companion to Linguistic Anthroplogy. Malden : Blackwell, 222-244.

Groupe de Fribourg (2012). Grammaire de la période. Berne : Peter Lang.

Hühn, P. et al. (éds). The Living handbook of narratology [en ligne]. Hamburg : Hamburg University, URL : http://www.lhn.uni-hamburg.de/, page consultée le 5 janvier 2020.

Jacoby, S., Ochs, E. (1995). Co-costruction : An introduction. Research on Language and Social Interaction. 28 (3), 171-183.

Jefferson, G. (1978). Sequential aspects of storytelling in conversation. Dans Schenkein J. (éd). Studies in the organization of conversational interaction. New York: Academic Press, 219-248. 37.

Lipiansky, E.-M. (1993). L'identité dans la communication. Communication et Langages, 97, 31-

Maingueneau, D. (2002). L'ethos, de la rhétorique à l'analyse de discours [en ligne]. Version raccourcie et légèrement modifiée de : Problèmes d'ethos. Pratiques, 113-114, URL :

http://dominique.maingueneau.pagesperso-orange.fr/pdf/Ethos.pdf, page consultée le 22 mai 2019, 118.

Maingueneau, D. (2013). L'èthos : un articulateur. COnTEXTES [en ligne], 13, URL : http://journals.openedition.org/contextes/5772, page consultée 16 avril 2019.

Marnette, S. (2002). Aux frontières du discours rapporté. Revue Romane, 37-1, 3-30.

Ochs, E. (2006 [2004]). Narrative lessons. In Duranti, S (ed.). A Companion to Linguistic Anthroplogy. Malden : Blackwell, 269-289.

Perrin, L. (1995). Du dialogue rapporté aux reprises diaphoniques. Cahiers de linguistique française, 16, 211-240.

Perrin, L. (2009). L'Ethos de l'oralité comme forme de fiction énonciative dans la presse écrite. Dans Burger, R., Jacquin, J., Micheli, R. (éds). Le Français parlé dans les médias : les médias et le 
politique. Actes de colloque, Lausanne. La version à laquelle nous nous rapportons est publiée en ligne avec une pagination indépendante, URL : https:/www.unil.ch/clsl/files/live/sites/cls1/files/shared/Actes FPM 2009/PerrinFPM2009.pdf, page consultée le 20 février 2019, 1-12.

Rosier, L. (1999). Le Discours rapporté. Histoire, théories, pratiques. Paris, Bruxelles : Duculot.

Rosier, L. (2008). Le Discours rapporté en français, Paris : Ophrys.

Tannen, D. (1984). Conversational style : analyzing talk among friends. Norwood : Ablex Publishing Corporation.

Tannen, D. (1989). Talking voices. Repetition, dialogue and imagery in conversational discourse. Cambridge : Cambridge University Press.

Vincent, D. (2004). Discours rapporté, représentations sociales et présentation de soi. Dans Munoz, J. M., Marnette, S., Rosier, L. (éds). Le Discours rapporté dans tous ses états. Actes du Colloque International, Bruxelles, 8-11 novembre 2001, 235-244. Éditeur.

Vincent, D., Dubois, S. (1997). Le Discours rapporté au quotidien. Québec : Nuit Blanche

Volochinov, V. N. (1977). Le Marxisme et la philosophie du langage. Essai d'application de la méthode sociologique en linguistique. Trad. du russe par Marina Yaguello. Paris : Minuit.

\section{Conventions de transcription}

\# : segment supprimé dans l'enregistrement

$\%$ : segment incompréhensible

$(0.2545)$ : pause en secondes

((rire)) : productions vocales

Crochets : chevauchements

Soulignement : DD

Soulignement pointillé : DInt

Tiret : amorce de construction

Référence : unine $15 \mathrm{~b} 03 \mathrm{~d}$

\section{Corpus}

Genre de parole : discussion

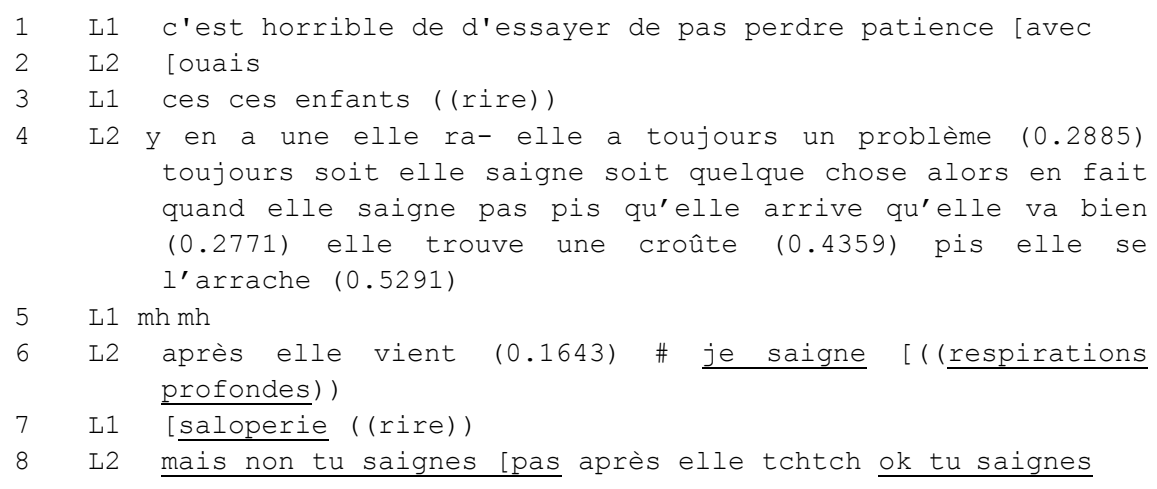


9 L1 [t'as le \% stock de] de pansements avec toi (0.2365) en fait tu le prends dans la salle (0.6951) tu [tu vois des croûtes

10 L2 [non on a-

11 L1 tu mets tes ((rire))

12 L2 on a la pharmacie tu sais du coup euh on a assez mais $(0.5279) \quad[\%$

13 L1 [faut demander] à ses parents tu sais (1.5672)

14 L2 mais j'ai déjà dit mais

15 L1 si elle vient ((rire)) vous mettez des sparadraps sur toutes ses croûtes

16 L2 on lui fait un spara- tu sais [genre euh \%

17 L1 [( (rire))] [momie ((rire))

18 L2 [avant d'entrer dans la salle] c'est une momie (0.9185) on la momifie à chaque fois (1.1825) ah non elle est terrible (1.0744)

19 L1 ouais [\%

20 L2 [pis] surtout quand elle voit que les autres euh (0.3513) ils ont un problème (0.5216) parce que si j-y en a un qui saigne ou qui a un souci pis qu'on s'occupe de lui direct elle va se trouver une croûte (0.3201) pis justement la dernière fois c'était sous son pantalon tu sais (0.4426) que vraiment elle vient vers moi eh regarde je saigne pis elle avait un trois-quarts $(0.6486)$ t'es là mais c'est au milieu de ta cuisse donc ça veut dire que tu as remonté ton pantalon tu as arraché ta croûte

21 L1 ah ouais d'accord

22 L2 p-t'es venue vers mois (0.3717) mais

$23 \mathrm{~L} 1 \mathrm{mh}$

24 L2 ((rire))

25 L1 bon

\section{Référence : unifr14a05d}

\section{Genre de parole : discussion}

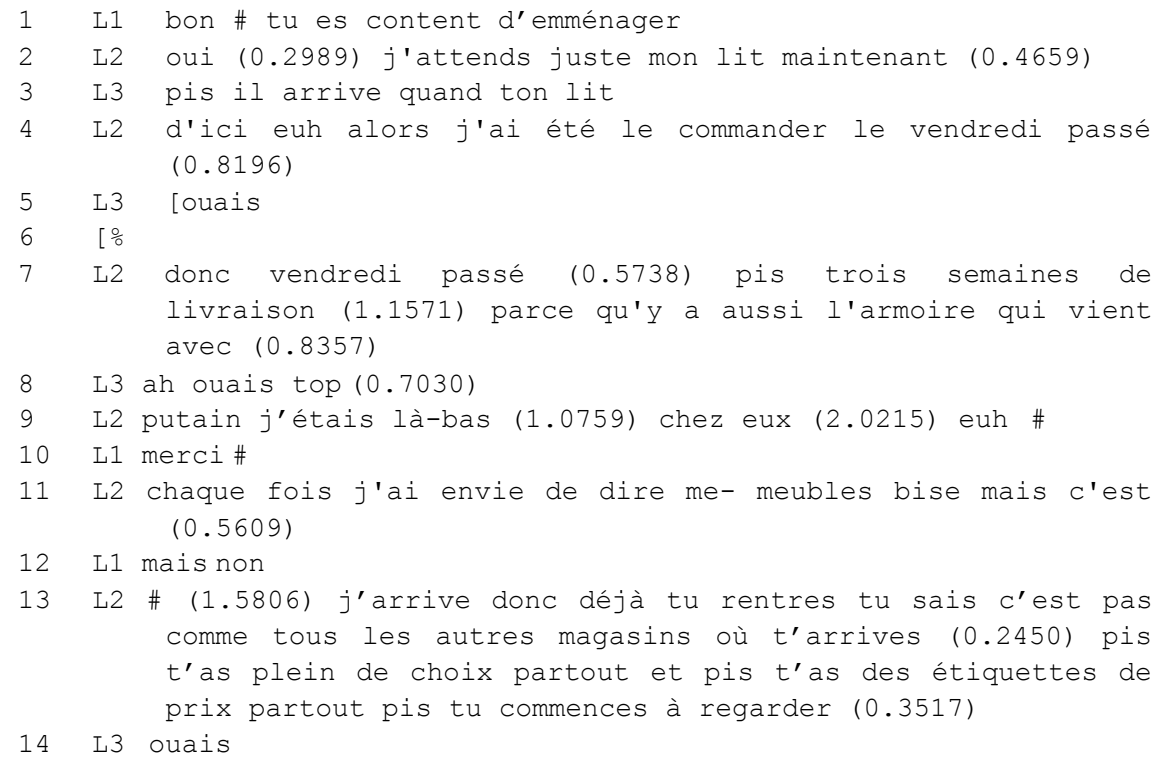


15 L2 pis personne s'occupe de toi (0.3807) non là t'arrives t'as une une une genre une secrétaire ou bien une réceptionniste qui a qui s'est levée $(0.3320)$ qui est venue vers nous (0.1660) euh j'étais avec \# (0.2324)

16 L1 bonjour v- allez prendre un café (0.7232)

17 L2 non [C'est euh

18 L3 [( (rire))

19 L2 bonjour je peux vous aider j'ai dit ouais euh (0.6043) on on vient regarder pour un lit alors un instant je vais chercher madame \# $(0.5048)$ la directrice qui arrive $(0.6621)$ vous êtes intéressés alors à avoir un lit euh alors venez on va on va aller regarder en bas (0.7738)

20 L1-L3 [( (rire))

21 L2 [\%] faire une espèce de de (1.6990)

22 L1 devisite

23 L2 pas vraiment de visite parce qu'elle nous a rien dit alors ça c'est tel modèle ça c'est tel modèle $(0.4981)$ on commence à tourner un peu pour voir les trucs pis après elle me dit ouais euh (1.3614) vous avez euh vous avez quel budget pour votre lit (1.2056)

24 L3 ( (rire))

25 L2 moi je venais de regarder les prix conforama ces prix euh promotion euh trois cent balles euh (0.6197)

26 L1 [complet

27 L2 [cadre] cadre sommier matelas monté livré donc euh je me suis dit bon

28 L3 (

29 L2 0.9384) je sais aussi que les lits ça peut aller plus haut (0.9163) je me dis allez (0.2192) je vais [assez haut

30 L1 [s-] surtout chez\#

\#

31 L2 je vais assez haut et je dis ouais mille deux cents mille deux cents francs euh cadre sommier matelas (0.6298)

32 L3 \% ça c'est les prix d'ikea non

33 L1 [( rire))

34 L2 [ouais

35 L3 mh $(0.6636)$

36 L2 C'est les prix ikea (1.6015)

37 L1-L3 ( (rire))

38 L2 enfin j'ai j'ai j'ai tapé entre ikea et conforama

39 L3 ( (rire))

40 L2 mais euh elle enlève ses lunettes d'un air dramatique tu sais ça faisait vraiment film euh limite tremblant pis elle fait (0.4051) bon (0.4383) mille deux cents francs vous comptez

41 L1 are you fucking kidding me

42 L3 [( (rire))

43 L2 [à peu] près mais dans d'autres termes (1.1270) elle a fait $(0.2458)$ mais $j-j e$ pourrai pas vous satisfaire

44 L3 ( (rire))

${ }^{1}$ Ce développement des deux sens d' « appropriated » vient de nous ; il nous semble que Tannen vise essentiellement le premier que nous décrivons.

${ }^{2}$ Et avant elle Volochinov (1977) ou Authier-Revuz (1978), entre autres. 
${ }^{3}$ Comme le décrit Perrin (1995 : 217), l'interprétation d'une séquence rapportée se déroule en fait en deux temps : "Pour comprendre un énoncé comme Pierre a dit ' $P$ ", il faut d'abord interpréter " $P$ ", non comme un énoncé du narrateur, pas même comme l'objet de ce qui est communiqué par ce dernier, mais comme un énoncé de Pierre proféré dans un contexte indépendant. C'est à cette seule condition que l'interprète sera ensuite à même d'en déduire ce que le narrateur communique à propos de Pierre en rapportant son propos dans un contexte immédiat. » Toutefois, étant donné que ce « contexte indépendant » n'a d'existence au moment de la profération du DD que dans et par le « contexte immédiat », on peut affirmer que c'est bien ce dernier qui est déterminant. Ce n'est pas tant ce qui a été fait par un discours au moment où il a été prononcé qui compte pour le $\mathrm{DD}$, que ce qui en est fait quand il est rapporté. C'est d'autant plus vrai que la conception du DD comme simple rapport ou répétition a été balayée depuis longtemps (voir par exemple Authier-Revuz, 1978, Tannen, 1989, Vincent \& Dubois, 1997 ou Rosier, 1999, 2008).

${ }^{4}$ À ce propos, voir Caillat (2013). Bien sûr, tout discours rapporté ne suppose pas un discours premier, qui serait répété plus ou moins fidèlement. Mais dans les différents cas où le discours ne relève pas d'une répétition (voir Vincent \& Dubois, 1997 ou Tannen, 1989), il est tout de même possible d'imaginer ou d'inférer à partir de connaissances du monde ou de l'état de la mémoire discursive (Groupe de Fribourg, 2012) qu'une certaine tonalité ou un certain contenu par exemple sont plus congruents ou plus probables que d'autres.

${ }^{5}$ C'est le cas typiquement lorsque le locuteur rapporté est un être animé non parlant, ou même un être inanimé : un nourrisson que son entourage fait parler, un animal de compagnie, ou un train comme dans cette situation à laquelle nous avons assistée, où un jeune homme s'est exclamé, alors que la locomotive entrait en gare, « Poussez-vous j'arrive ! »

${ }^{6}$ C'est une idéalisation, mais qui n'est pas sans fondement rationnel. Les chevauchements rendent tout simplement la compréhension difficile. Mais ils peuvent par ailleurs être parfaitement admis par exemple dans certains " conversational styles », d'après Tannen (1984). Cette chercheuse analyse même les chevauchements fréquents comme un trait caractéristique du style à « high-involvement ».

${ }^{7}$ Charles et Marjorie Goodwin définissent la «participation » comme les « actions demonstrating forms of involvement performed by parties within evolving structures of talk » (2004 : 222).

${ }^{8}$ Notons bien que l'usage « stratégique » du langage ne suppose pas forcément une mobilisation entièrement consciente et intentionnelle des moyens langagiers par le sujet parlant.

${ }^{9}$ Ochs a bien noté cette possibilité d'une co-narration active du récit par plusieurs participants. Elle inscrit cette tendance dans ce qu'elle appelle la " Narrative Practice 2 », opposée sur de multiples dimensions (type de narration, racontabilité, relation à l'activité en cours, intrigue et positionnement moral) à la « Narrative Practice $1 »($ Ochs, 2006). Les « narratives of personal experience » relèvent selon elle de l'une ou de l'autre pratique, avec bien sûr des degrés multiples entre ces deux pôles.

10 C. et M. Goodwin adressent selon nous au modèle goffmanien du « footing » des critiques dirimantes, en même temps qu'ils élaborent un cadre permettant de le dépasser. Voir par exemple leur contribution de 2004 au Companion to Linguistic Anthropology.

${ }^{11}$ C'est-à-dire un protagoniste ou un personnage d'une scène décrite (Goffman, 1981 : 147).

${ }^{12}$ C'est-à-dire la « sounding box in use » ou « talking machine » (Goffman, 1981 : 144) de l'énoncé en question.

13 À propos de la définition et du fonctionnement de ce concept, voir notamment Maingueneau 2002 , 2013, Perrin, 2009 ou Amossy, 2010.

${ }^{14}$ Goffman (1982 : 113) allait jusqu'à décrire ainsi la conversation dans laquelle deux individus sont pleinement engagés : " As a main focus of attention talk is unique, however, for talk creates for the participant a world and a reality that has other participants in it. Joint spontaneous involvement is a unio mystico, a socialized trance. »

${ }^{15} \mathrm{La}$ 《 racontabilité » désigne tout ce qui peut rendre un récit digne d'être relaté. Pour plus de précisions, voir la notice « tellability » sur le site du Living handbook of narratology, URL : http://www.lhn.uni-hamburg.de/node/30.html, page consultée le 5 janvier 2020.

${ }^{16}$ Elle consiste pour les auteurs à « mettre dans la bouche de quelqu'un (ou de soi-même) des propos qui n'ont explicitement jamais été dits » (Vincent \& Dubois, 1997 : 61).

${ }^{17}$ Nous nous référons pour l'analyse de ce marqueur à Beeching (2007).

${ }^{18}$ Les extraits peuvent être écoutés sur OFROM. La référence exacte est indiquée en annexe. 
${ }^{19}$ L'expression « are you fucking kidding me » apparaît sous de nombreuses formes et sur divers supports ou médiums, donnant lieu par exemple à d'innombrables GIFs. Elle correspond également à un mème que le site Know Your Meme décrit comme « a rage comic character wearing a scornful facial expression that is typically used in relation to someone else's extreme stupidity » (URL : https://knowyourmeme.com/memes/are-you-fucking-kidding-me, page consultée le 30 décembre 2019).

${ }^{20}$ Le changement de langue a peut-être été encouragé par la comparaison cinématographique de L2 auparavant (ça faisant vraiment film, au TP 40), mais nous l'expliquons plutôt par la disponibilité cognitive et socio-culturelle de l'expression choisie en anglais, ainsi qu'à son efficacité rhétorique. 